\title{
Une proposition de caractérisation de l'émotion sportive : perception des indices émotionnels dans le commentaire sportif en direct
}

\author{
Carolyn Fontagnol ${ }^{1}$, Catherine Mathon ${ }^{2}$ \\ ${ }^{1}$ FoReLLIS (EA3816), Université de Poitiers \\ ${ }^{2}$ CLLE-ERRSSàB (UMR5362), Université Bordeaux Montaigne
}

\begin{abstract}
Résumé. Le commentaire sportif télévisé en direct (CSTD) est un genre contraint (contraintes de médium, de contenu, de genre) qui présente des spécificités linguistiques (dialogue voix/images, grande proportion de phrases averbales, patrons prosodiques spécifiques). Souhaitant nous intéresser aux manifestations linguistiques de l'émotion dans le CSTD, nous présentons dans cette étude un protocole de recherche basé sur la question de l'émotion, adapté à la nature de notre corpus. A partir de matchs de rugby de la coupe du monde 2007 et 2015, nous présentons les résultats des deux tests de perception qui nous ont permis, d'une part, d'identifier et caractériser l'émotion dans le commentaire sportif télévisé en direct, d'autre part, de déterminer l'importance du matériel prosodique dans la caractérisation de l'émotion dans ce type de corpus.
\end{abstract}

\begin{abstract}
A proposition for the characterization of sport emotion: perception of emotional features in TV sport commentary. TV sport commentary is a genre of discourse which is produced under different constraints (from the medium, from the content that is reported, from the genre itself) and which presents linguistic specificities such as an important proportion of nonverbal phrases or specific melodic patterns. This paper is interested in the linguistic expressions of sport emotion in TV sport commentary. Thus, we present is this study an experimental protocol built up in order to question the presence of sport emotion in a corpus of TV sport commentaries selected from matches of the Rugby World Cup 2007 \& 2015. Based on two perceptual tests, our results show first that it is possible to identify and characterize sport emotion, second that prosodic information is particularly relevant in the detection of sport emotion.
\end{abstract}

\section{INTRODUCTION}

Le travail que nous présentons ici s'inscrit dans la continuité des études menées d'une part sur les genres de discours oraux et plus particulièrement sur un phonogenre particulier qui est le commentaire sportif en direct, et d'autre part sur l'expression linguistique et prosodique des émotions, affects, attitudes.

Le commentaire sportif en direct présente beaucoup de spécificités linguistiques qui en font un objet de recherche riche et productif à différents niveaux :

- Lié aux images qu'il commente, quand il est télévisuel, il pose la question de l'iconicité de la parole (Mathon \& Boulakia, 2009), ou tout du moins des ajustements du discours aux images ;

- Du point de vue syntaxique, il présente une forte proportion de phrases averbales et des formes d'énoncés spécifiques qui semblent s'adapter aux périodes du jeu (Deulofeu, 2000 ; Hartmann, 2011 ; Augendre et al., 2018);

- Du point de vue prosodique, le commentaire sportif en direct présente des patrons prosodiques qui lui sont propres (Audrit et al., 2012). Le rythme de parole du journaliste sportif semble corrélé avec celui du jeu, au moins dans certaines phases du discours (Mathon et al., 2016).

Les spécificités linguistiques du commentaire sportif en direct sont liées aux contraintes qui pèsent sur ce phonogenre (Mathon et al., 2015) :

\footnotetext{
${ }^{1}$ Corresponding author : carolyn.fontagnol@univ-poitiers.fr
} 
Les contraintes de medium et de contenu impactent indéniablement les structures linguistiques (Augendre et al., 2018) mais aussi la construction même du discours en périodes de "play-by-play», de "colorcommentary » (Hartmann, 2011) et de « supportaire » (Mathon et al., 2015);

Les contraintes de genre, à la fois journalistique et médiatique, vont influencer la présence de subjectivité dans le discours et vont encourager l'expression de « l'émotion sportive » (Augendre et al., 2014).

L'existence d'une émotion sportive, exprimée par les journalistes sportifs ou ressentie par le téléspectateur, est parfaitement reconnue et acceptée. Elle semble faire écho aux émotions exprimées par les sportifs euxmêmes (Lévêque, 2014). Pour autant, elle n'a fait que très peu l'objet d'études en linguistique, alors même que les travaux sur l'expression des émotions et attitudes sont très nombreux et constituent un champ de recherche très dynamique (voir sur la question de la prosodie émotionnelle par exemple l'état de l'art proposé par Bąk (2016)).

Nous proposons donc dans cette étude de caractériser l'émotion ou les émotions sportives, en utilisant des tests de perception basés sur des protocoles issus du champ de la psychologie. Le premier test de perception a pour objectif de confirmer ou d'infirmer la présence d'une émotion sportive exprimée dans le commentaire et de repérer sur quels segments du discours en lien avec le jeu l'émotion est la mieux reconnue. En effet, Mathon et al. (2015) font l'hypothèse que la présence d'émotion dans le commentaire sportif en direct est sujette aux enjeux de la rencontre sportive et que les actions qui vont permettre de marquer des points sont également celles qui seront le plus colorées affectivement. Il s'agit également de vérifier que les outils traditionnels mis en œuvre pour la reconnaissance de l'émotion, au sens classique du terme, sont efficaces également pour l'émotion sportive. Le second test de perception affine les outils et les protocoles expérimentaux déjà mis en œuvre et pose la question du poids des indices acoustiques dans la reconnaissance de l'émotion.

\section{LE CORPUS}

L'ensemble de cette étude repose sur un large corpus multimodal et multilingue de commentaires sportifs télévisuels en direct (désormais CSTD) :

- $\quad$ Il est multimodal car constitué du son mais également des images, et celles-ci font l'objet d'annotations au même titre que le commentaire qui les accompagne ;

- Il est multilingue car chaque enregistrement vidéo fait l'objet d'un commentaire en français et dans une autre langue (anglais ou japonais). Il s'agit donc à ce titre d'un corpus oral comparable, puisque des images identiques sont commentées dans deux langues différentes.

Le corpus est constitué d'enregistrements de matchs télévisés des coupes du monde 2007, 2015 et, ajout récent, 2019 de rugby à XV. Ces matchs sont tous commentés en français et dans une autre langue, principalement l'anglais. Quelques matchs de la coupe du monde 2007 (Lortal \& Mathon, 2008) ainsi que de la coupe du monde 2019 font l'objet d'un commentaire en japonais.

Dans le cadre des deux expérimentations que nous avons menées, le corpus de travail a été réduit à 4 matchs de la coupe du monde de rugby 2015 commentés en français en ce qui concerne le premier test de perception, et 1 match de la coupe du monde 2007 commenté en français pour le second test de perception.

\section{UN TEST DE PERCEPTION POUR CARACTERISER L'EMOTION}

Afin de ne pas nous fier à notre propre jugement dans l'identification de l'émotion dans le CSTD, nous avons créé un test de perception ciblé sur des tâches de caractérisation de l'émotion (Fontagnol et al., 2018). Ce test de perception est un pré-test que nous avons mis en place en vue de tester les outils de mesure de l'émotion que nous avons emprunté au domaine de la psychologie et adapté à une étude en linguistique.

\subsection{Protocole expérimental}

\subsubsection{Sélection des stimuli}

À partir d'une revue de presse constituée d'articles issus de sites spécialisés dans l'actualité du sport: L'équipe (www.lequipe.fr) et Rugbyrama (https://www.rugbyrama.fr), nous avons d'abord sélectionné des matchs dont l'enjeu de la rencontre et le sémantisme des qualificatifs donnés aux rencontres dans la presse écrite était particulièrement subjectif et intense. Nous avons surveillé les titres évocateurs construits à partir de noms et d'adjectifs subjectifs tels que " domination », " humiliation », " incroyable », " fantastique », autrement dit des titres dont le sémantisme était caractérisé par une valence positive ou négative et une intensité forte. Les matchs retenus pour le test de perception ont été les suivants : Australie/Nouvelle-Zélande (match final, élimination de l'Australie) ; France/Nouvelle-Zélande (match de quart, élimination de la France) ; Angleterre/Australie (match de poule, élimination de l'Angleterre). Par la suite, et en vue de tenir compte de l'impact des images de jeu sur 
les manifestations linguistiques de l'émotion dans le discours, nous avons pris appui sur les «minutes » de jeu (commentaire en ligne en quasi-direct du match sportif) afin de repérer les actions de jeu proéminentes dans le match. Nous avons ainsi élaboré une pré-classification des extraits en fonction des cinq types d'actions suivants

- $\quad$ Action décisive $(\mathrm{AD})$ : une action décisive aboutit à un essai marqué par l'un des joueurs.

- Action collective (AC) : une action collective est caractérisée par un jeu offensif, il s'agit d'une action qui permet de gagner du terrain vers la zone d'en-but adverse.

- Action transformation (AT) : une action de transformation après un essai permet de gagner deux points supplémentaires.

- Action manquée (AM) : une action manquée lorsque l'une des deux équipes commet une faute ou qu'une occasion de gagner du terrain vers la zone d'en-but adverse n'est pas saisie.

- Commentaire neutre $(\mathrm{CN})$ : commentaire hors direct de type anecdotique portant sur un joueur ou une action ne présentant aucun enjeu pour l'issue du match. Du fait que les extraits de ce groupe sont a priori opposés aux autres stimuli de par leur nature hors-direct et du fait qu'ils renvoient à des moments sans enjeu véritable pour le match, nous considérons que le groupe "CN" est un groupe contrôle.

Pour chaque catégorie, nous avons extrait cinq stimuli dans les trois matchs d'une durée variant de dix à vingt secondes.

\subsubsection{Les deux outils de mesure}

Afin de mesurer l'émotion perçue dans les stimuli, nous avons proposé aux participants de caractériser l'émotion à l'aide de deux outils. Le premier outil est très utilisé dans les études en psychologie sur l'émotion, il s'agit du Self-Manikin Assessment (Bradley \& Lang, 1994) et il permet aux participants d'attribuer une valeur émotionnelle à un stimulus en fonction de différentes mesures. Dans cette étude, l'émotion est caractérisée en fonction des mesures de la valence et de l'intensité. Cet outil non-verbal présente l'avantage de pouvoir caractériser l'émotion sans faire appel à une étiquette verbale, et donc de ne pas influencer la perception du participant. Sur les figures ci-dessous, on observe que les étiquettes différent selon cinq degrés représentés de gauche à droite par des bonhommes du plus au moins souriant pour la valence (figure 1), et du plus petit au plus grand pour représenter l'intensité (figure 2) :

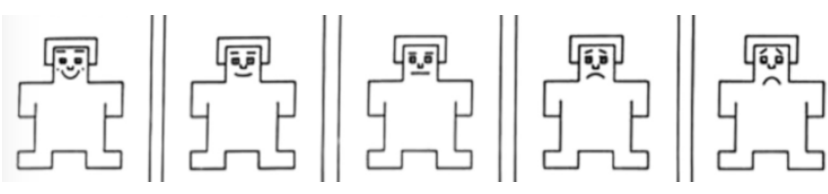

Figure 1 : Échelle correspondant à la valence dans le SAM

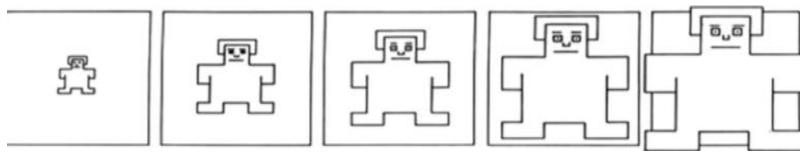

Figure 2 : Échelle correspondant à l'intensité dans le SAM

Le second outil est un outil verbal également très utilisé dans les études en psychologie sur les émotions, il s'agit du Brief Mood Introspection Scale (BMIS) développé initialement par Mayer \& Gaschke et traduit en français par Niedenthal \& Dalle (2001) : 


\begin{tabular}{|c|c|c|c|c|}
\hline & Pas du tout & & & Tout à fait \\
\hline 1. Dynamique & XX & $\mathrm{X}$ & V & VV \\
\hline 2. Heureux & $\mathrm{XX}$ & $\mathrm{X}$ & V & VV \\
\hline 3. Triste & XX & $\mathrm{X}$ & V & VV \\
\hline 4. Fatigué & $\mathrm{XX}$ & $\mathrm{X}$ & V & VV \\
\hline 5. Bienveillant & $\mathrm{XX}$ & $\mathrm{X}$ & V & VV \\
\hline 6. Content & $\mathrm{XX}$ & $\mathrm{X}$ & V & VV \\
\hline 7. Mélancolique & $\mathrm{XX}$ & $\mathrm{X}$ & V & VV \\
\hline 8. Excité & $\mathrm{XX}$ & $\mathrm{X}$ & V & VV \\
\hline 9. Épuisé & $\mathrm{XX}$ & $\mathrm{X}$ & V & VV \\
\hline 10. Grincheux & $\mathrm{XX}$ & $\mathrm{X}$ & V & VV \\
\hline 11. Énergique & XX & $\mathrm{X}$ & V & VV \\
\hline 12. Nerveux & $\mathrm{XX}$ & $\mathrm{X}$ & V & VV \\
\hline 13. Calme & $\mathrm{XX}$ & $\mathrm{X}$ & V & VV \\
\hline 14. Affectueux & XX & $\mathrm{X}$ & V & VV \\
\hline 15. Agacé & $\mathrm{XX}$ & $\mathrm{X}$ & V & VV \\
\hline 16. Vif & $\mathrm{XX}$ & $\mathrm{X}$ & V & VV \\
\hline
\end{tabular}

Figure 3: Liste des seize adjectifs émotionnels issus du BMIS

Cet outil est construit quant à lui à partir d'un set de seize adjectifs émotionnels (figure 3) auxquels sont associés quatre valeurs allant de "pas du tout" à "tout à fait".

\subsubsection{Les conditions de passation du test}

Quatorze participants ont accepté de participer à ce test et de mesurer l'émotion dans les vingt-cinq extraits présentés. Les quatorze participant.e.s (huit femmes et six hommes) constituent un groupe dont le niveau d'étude se situe entre bac +3 et bac +8 . La moyenne d'âge des participant.e.s est de vingt-cinq ans et tous sont locuteurs du français. La passation s'est déroulée dans un boxe spécifique aux expérimentations en laboratoire de manière individuelle. Le déroulement du test s'est fait en deux parties, dans un premier temps les stimuli ont été présentés et l'émotion a été annotée à partir du SAM et dans un second temps, à partir du BMIS. Les stimuli étaient présentés aléatoirement et la consigne donnée était de répondre le plus instinctivement possible.

\subsection{Les résultats et la discussion}

\subsubsection{Les résultats du SAM}

Afin de représenter les résultats du SAM, nous avons choisi une représentation en nuage de points. Sur la figure 4, chaque point représente un individu et cette répartition se fait en fonction des réponses obtenues aux mesures de la valence et de l'intensité, pour chaque extrait. L'axe horizontal qui correspond à celui de l'intensité, s'oppose à l'axe vertical qui correspond à celui de la valence. Bien que l'on puisse observer une certaine dispersion dans les résultats, trois groupes émergent en accord avec la pré-classification opérée en types d'action. Les groupes qui émergent sont les suivants:

- le groupe "AD" est représenté en haut à droite de la figure et mis en évidence par un rond noir au trait épais. On observe que ce groupe se caractérise notamment par une intensité forte et une valence positive.

- le groupe "CN" est représenté au centre à gauche et mis en évidence par un trait noir en pointillé. Ce groupe est caractérisé par une intensité basse à moyenne et une valence moyenne.

- le groupe "AM" est représenté en bas à droite de la figure et mis en évidence par un triangle au trait noir épais. Ce groupe est caractérisé par une intensité plutôt forte et une valence négative. 


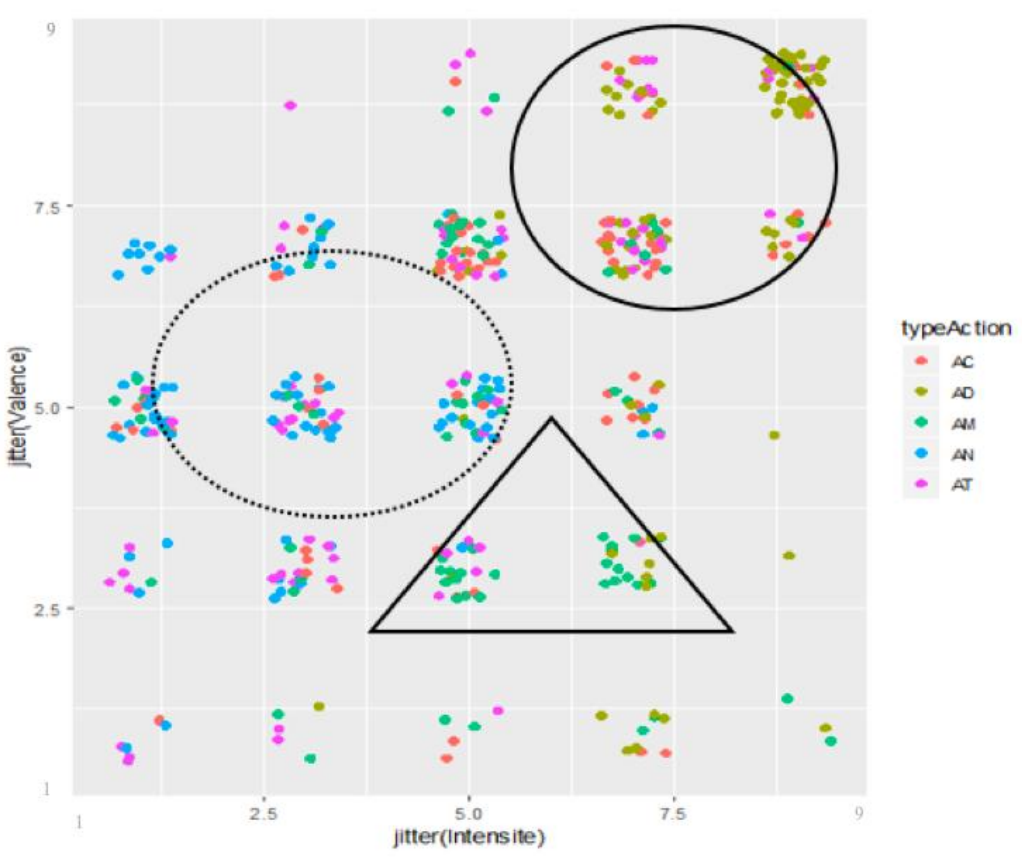

Figure 4 : Représentation des individus en fonction des deux mesures : valence et intensité

La dispersion observée dans les résultats laisse penser que l'association des deux mesures "valence" et "intensité" n'est pas la plus adéquate à notre étude et qu'une troisième mesure pourrait être nécessaire afin de distinguer les groupes "AC" et "AT" qui faisaient partie de notre pré-classification.

\subsubsection{Les résultats du BMIS}

Contrairement aux résultats obtenus avec le SAM, le grand nombre de mesures (pour rappel, seize adjectifs émotionnels) a rendu difficile la phase d'exploration des données. Afin de visualiser l'information principale obtenue avec le BMIS, nous avons mis en place une ACP (analyse en composantes principales) ayant pour objectif de condenser l'information en fonction d'un certain nombre d'axes qui se veulent représentatif des tendances observables dans le corpus.

L'analyse selon le premier plan factoriel ne représente que 57,7\% de l'information et met en évidence la pertinence de certaines mesures pour la caractérisation de l'émotion dans le CSTD. Nous observons notamment que de même que pour les résultats du SAM, trois groupes émergent en accord avec la pré-classification (figure 4):

- le groupe “AD” est mis en évidence par un rond noir au trait épais. Ce groupe est caractérisé par les adjectifs "dynamique", "énergique", "excité", "vif", "heureux" et "content";

- le groupe "CN" est mis en évidence par un rond noir en pointillé. Ce groupe est caractérisé par l'adjectif "calme";

- le groupe "AM" est représenté par un triangle noir. Bien que les individus soient dispersés, on observe que les réponses sont structurées autour des adjectifs "nerveux" et "agacé" par opposition aux adjectifs "bienveillant" et "affectueux". 


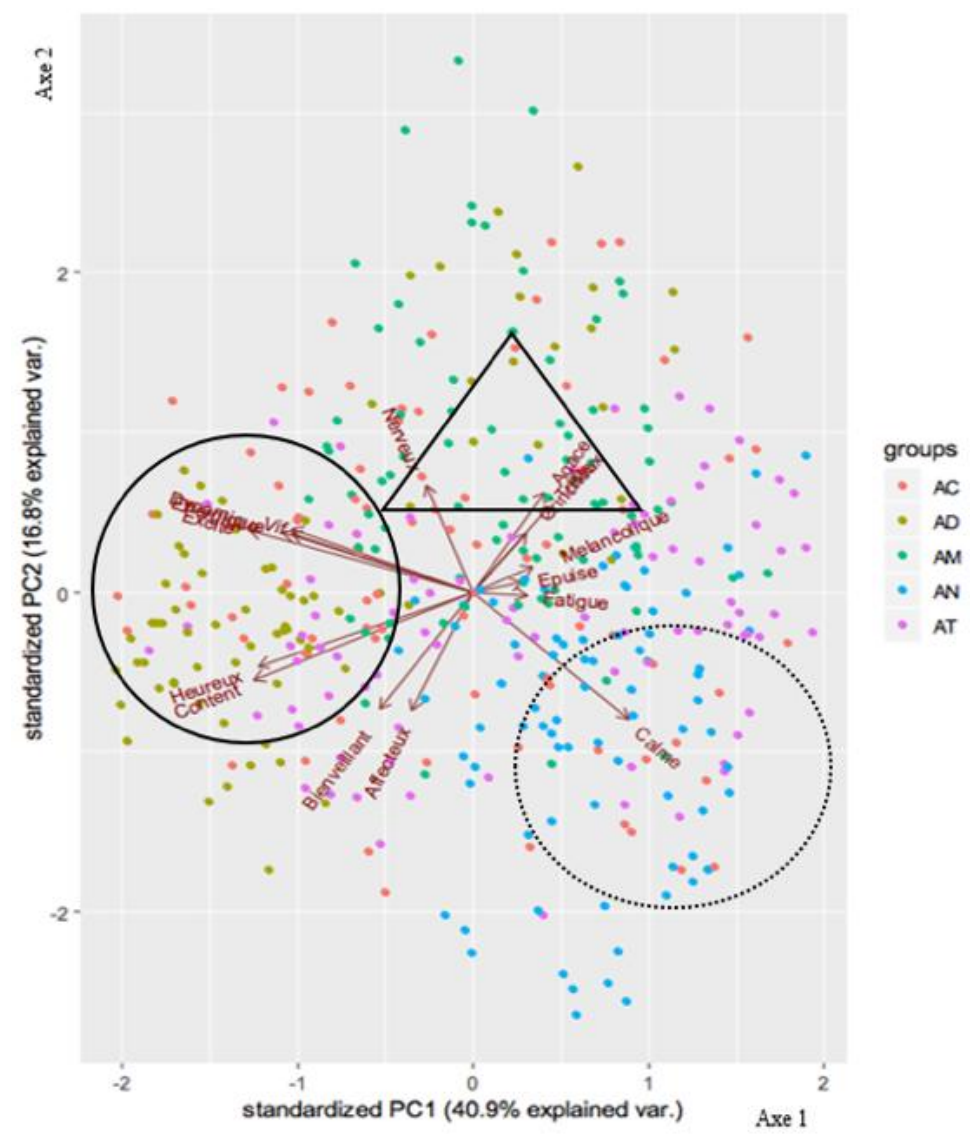

Figure 5 : Visualisation du premier plan factoriel des résultats du BMIS

Le test du BMIS permet de mettre en évidence que les seize adjectifs ne sont pas tous pertinents pour la description de l'émotion dans les stimuli. Par ailleurs, parmi les adjectifs pertinents pour la caractérisation de l'émotion, nous observons des redondances sémantiques. Par exemple pour le groupe AD, les redondances se situent au niveau des adjectifs renvoyant à l'intensité (ex: "dynamique", "vif", "énergique" et "excité") ou à la valence (ex: "heureux" et "content").

\subsubsection{Discussion}

Pour ce premier test de perception nous avons fait appel à deux outils de mesure de l'émotion. L'objectif de cette démarche était, d'une part, de ne pas influencer les participants avec des étiquettes émotionnelles, d'autre part, de ne pas nommer nous-mêmes les émotions qui caractérisent nos stimuli. A l'issue des analyses statistiques, nous observons une convergence dans les résultats des deux tests puisque pour chaque test, les trois groupes "AD", "CN" et "AM" émergent en accord avec la pré-classification. Notre groupe contrôle "CN" s'oppose notamment au groupe “AD" et au groupe “AM" caractérisés par une valence positive pour l'un et négative pour l'autre, et une intensité forte. Nous faisons l'hypothèse qu'il manque à notre test une variable qui permettrait de distinguer les groupes "AT" et "AC".

\section{UN TEST DE PERCEPTION POUR DETERMINER L'IMPORTANCE DE LA PROSODIE DANS LA DETECTION DE L'EMOTION}

Le premier test de perception a montré que les outils traditionnels de détection et de caractérisation de l'émotion pouvaient être appliqués sur des extraits audio de CSTD dans le but de définir l'émotion sportive. Ce premier test de perception nous a permis d'affiner notre protocole expérimental, afin de le rendre plus efficace. Les résultats du BMIS, notamment du fait du grand nombre d'étiquettes désignant des émotions, ont été relativement difficiles à exploiter. Par ailleurs, nous ne savons pas dans quelle mesure les adjectifs choisis de manière prédéfinie influencent le jugement des sujets sur la nature de l'émotion sportive, alors même que celle-ci n'est justement pas définie.

Ainsi, nous avons préféré opté pour le second test pour une évaluation sur trois échelles (de type SAM) en incluant cette fois-ci l'excitation, en plus de la valence et de l'intensité. En effet, l'excitation est une échelle 
importante pour la détection des émotions classiques (colère, joie, tristesse, peur et dégoût). Par ailleurs, plusieurs choix d'étiquettes du BMIS dans le test précédent semblaient indiquer que cette dimension pouvait s'avérer importante. Enfin, un travail d'annotation des émotions sur le même corpus produit dans le cadre d'une autre étude ${ }^{i}$ suggérait que l'excitation était une caractéristique prépondérante du discours du CSTD.

Ce second test de perception s'appuie donc sur le premier pour affiner le protocole expérimental, mais par ailleurs il cherche à explorer d'autres hypothèses. Ainsi, non seulement s'agit-il de tester les outils de la psychologie pour caractériser l'émotion sportive, mais il s'agit également de vérifier l'importance de la prosodie dans la détection de l'émotion dans le CSTD. Ce second test porte donc sur des stimuli dont l'information lexicale a été neutralisée afin de tester l'impact de la seule information prosodique sur la reconnaissance de l'émotion sportive.

\subsection{Protocole expérimental}

\subsubsection{Sélection des stimuli}

A partir des actions marquantes recensées dans le résumé des minutes de jeu sur le site Rugbyrama ${ }^{\mathrm{ii}}$, nous avons repéré et délimité des stimuli sur la ligne temporelle du match Japon-Fidji de la Coupe du Monde 2007. Pour ce second test de perception, les stimuli ont été sélectionnés non pas en accord avec la pré-classification présentée dans la partie précédente (section 1.1.a), mais sur la base des actions proéminentes directement en lien avec une action de jeu particulière ayant lieu au cours du match. Les stimuli renvoient ainsi aux actions spécifiques suivantes : essai, transformation, pénalité, jeu offensif.

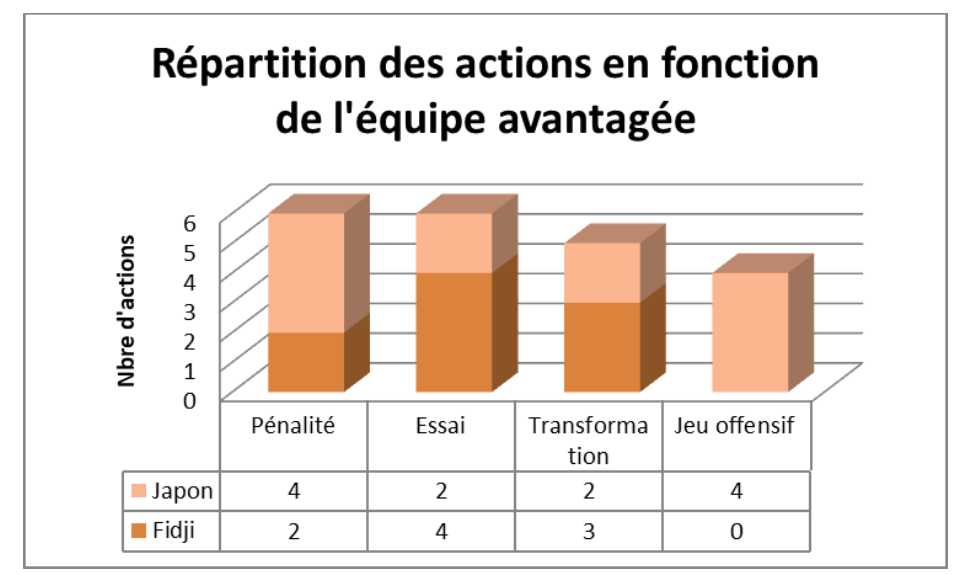

Figure 6: Répartition des actions en fonction de l'équipe avantagée.

On observe sur la figure 6 que les pénalités et le jeu offensif sont plus japonais, tandis que les essais et les transformations sont plus fidjiens. Il y a au total plus d'actions à l'avantage des japonais que des fidjiens, même si ces derniers gagnent la compétition 35 à 31. A partir de ces actions, trois sets de 21 stimuli audio ont été extraits du match Japon-Fidji dans des modalités différentes:

- 1 set de stimuli en japonais ;

- $\quad 1$ set de stimuli en français ;

- 1 set de stimuli filtrés afin de masquer le contenu lexical.

Pour la création des stimuli de ce deuxième test de perception, nous avons procédé à l'extraction automatique des stimuli japonais et français à partir des temps de début et de fin des actions. En ce qui concerne le troisième set, les stimuli ont été filtrés à l'aide du logiciel Praat afin de masquer l'information lexicale (Low-pass filtering, $500-1000 \mathrm{~Hz}$ ) : concrètement, il s'agit d'éliminer l'information acoustique sur une plage de fréquence donnée. A l'écoute, le sujet perçoit le rythme et la fréquence fondamentale (le contour mélodique), mais le signal semble trop lointain pour que les mots soient précisément perceptibles. Ce système de filtrage impacte toutefois l'intensité perçue du signal et pourrait avoir un impact également sur l'évaluation de l'émotion en termes d'intensité.

\subsubsection{Mesure de l'émotion}

Pour ce test, nous avons conservé les mesures de la valence et de l'intensité et avons ajouté celle de l'excitation. Les questions posées aux participants afin de les guider dans la caractérisation de l'émotion dans les différents sets de stimuli sont les suivantes: 
pas excité $=1<\longrightarrow 5=$ très excité

Vous devez répondre sur une échelle de 1 à 5 :

1 = si vous considérez que le locuteur ne manifeste aucune excitation ;

2 = si vous considérez que le locuteur manifeste une excitation faible;

3 = si vous considérez que le locuteur manifeste une excitation moyenne;

4 = si vous considérez que le locuteur manifeste une excitation forte ;

5 = si vous considérez que le locuteur manifeste une excitation très forte.

2. D'après vous, l'émotion que le locuteur exprime est...

très négative $=-2<\longrightarrow+2=$ très positive

Vous devez répondre sur une échelle de -2 à +2 :

-2 = si vous considérez que le locuteur exprime une émotion très négative ;

-1 = si vous considérez que le locuteur exprime une émotion négative ;

$0=$ si vous considérez que le locuteur est plutôt neutre ;

$+1=$ si vous considérez que le locuteur exprime une émotion positive ;

$+2=$ si vous considérez que le locuteur exprime une émotion très positive.

3. D'après vous, le locuteur exprime une émotion...?

très faible $=1<\longrightarrow$ très forte

1 = si vous considérez que le locuteur exprime une émotion très faible ;

2 = si vous considérez que le locuteur exprime une émotion faible ;

3 = si vous considérez que le locuteur exprime une émotion moyenne ;

4 = si vous considérez que le locuteur exprime une émotion forte ;

5 = si vous considérez que le locuteur exprime une émotion très forte.

Pour nous assurer que les sujets fassent bien la différence entre l'excitation et l'intensité, nous donnons l'exemple d'une émotion telle que la tristesse, qui peut être perçue comme intense sans pour autant exprimer aucune excitation.

\subsubsection{Conditions de passation}

Le test est proposé sur une plateforme de e-learning de type Moodle à une population d'étudiants francophones natifs pour la plupart, avec une moyenne d'âge de 20 ans. La population est divisée en deux groupes d'une vingtaine de sujets chacun. Chaque groupe teste une condition :

- $\quad$ le groupe FF évalue les stimuli en français filtrés ;

- $\quad$ le groupe $\mathrm{J}$ évalue les stimuli en japonais (On demande aux membres de ce groupe s'ils parlent ou sont en cours d'apprentissage du japonais).

Par ailleurs, un groupe contrôle évalue les stimuli en français avec accès à l'information lexicale.

Le test, composé de 21 questions (avec pour chaque stimulus trois sous-questions correspondant aux trois échelles) est présenté sous la forme d'un quizz au format xml moodle. Il dure environ une dizaine de minutes. Les consignes données aux sujets sont de faire le test en une seule passation, dans un endroit calme et avec des écouteurs, afin de garantir des conditions optimales pour la passation du test. Les réponses au test sont récupérées dans un fichier csv directement disponible à partir de la plateforme Moodle. La durée de passation du test est indiquée pour chaque sujet. Les réponses de sujets qui n'auraient pas respecté les consignes (au moins en ce qui concerne le temps) ne sont pas prises en compte. 


\subsection{Résultats}

Nous comparons dans cette section les résultats obtenus dans les deux conditions de passation du test sans accès au lexique (stimuli en français filtrés et stimuli en japonais) ainsi que ceux du groupe contrôle (stimuli en français) pour les trois échelles, excitation, valence et intensité.
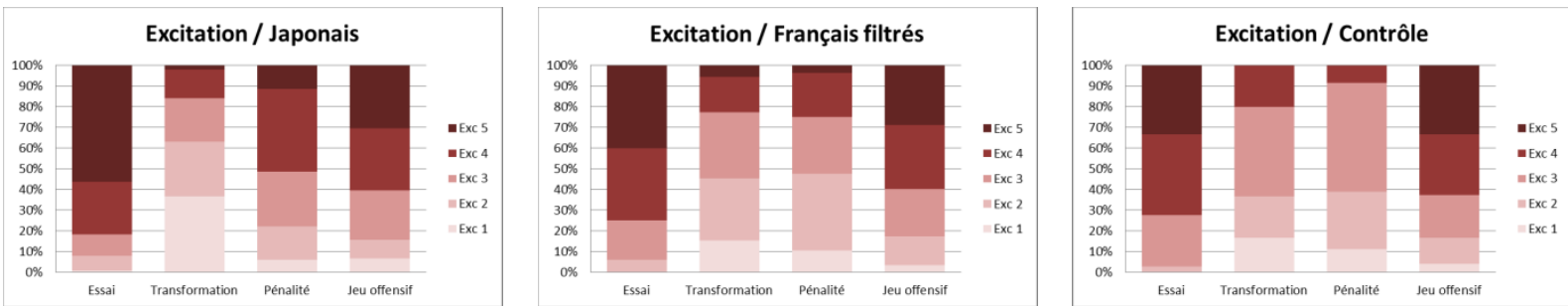

Figure 7 : Evaluation de l'excitation par les trois groupes (groupe J, groupe FF, groupe Contrôle) pour chacun des quatre types d'actions de jeu (essai, transformation, pénalité et jeu offensif).

La figure 7 ci-dessus montre les résultats obtenus pour l'évaluation de l'excitation pour les trois groupes. Il est intéressant de noter dans un premier temps que l'essai et le jeu offensif se détachent des deux autres comme étant les actions qui sont perçues avec les plus hauts niveaux d'excitation (E4 et E5). Si pour le jeu offensif, l'évaluation est sensiblement la même d'un groupe à l'autre, les niveaux 4 et 5 d'excitation représentant $60 \%$ des jugements, pour l'essai le groupe $\mathrm{J}$ a perçu des niveaux d'excitation élevés à $80 \%$. Ce résultat pourrait sans doute être mis en relation avec la question de l'émotion partisane (cf. 4.3. Discussion). Le groupe J se démarque également des deux autres groupes en ce qui concerne l'évaluation de l'excitation pour la pénalité, aux niveaux 4 et 5 pour $50 \%$ des jugements environ. Ceci peut sans doute être expliqué par le fait que le Japon a marqué beaucoup de points avec des pénalités réussies, ce qui donne à cette action une importance particulière dans le cadre de ce match.

La figure 8, ci-dessous, expose les résultats obtenus en ce qui concerne l'évaluation de la valence. Dans les trois groupes, l'essai, la pénalité et le jeu offensif sont perçus avec une valence positive à au moins $50 \%$. C'est l'essai qui obtient les niveaux les plus importants de valence positive (70 à $80 \%$ des jugements). Le groupe contrôle qui a accès à l'information lexicale aussi bien qu'à l'information prosodique évalue les différentes actions avec les plus hauts niveaux de valence positive. On peut émettre l'hypothèse que l'accès au contenu lexical permet peut-être de mieux évaluer la valence. On pourrait aussi considérer que les commentateurs français d'un match qui n'implique pas l'équipe nationale ont tendance à colorer plus positivement toutes les actions du match, quelle que soit l'équipe qui marque.
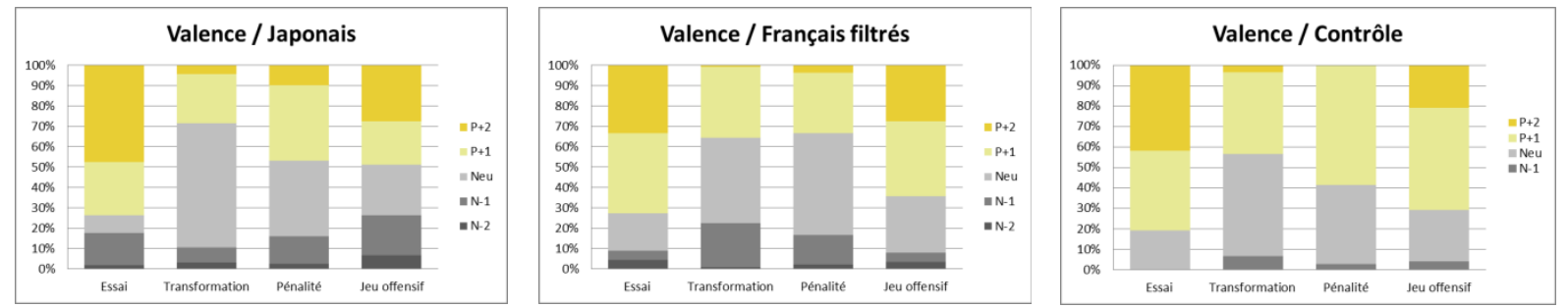

Figure 8 : Evaluation de la valence par les trois groupes (groupe J, groupe FF, groupe Contrôle) pour chacun des quatre types d'actions de jeu (essai, transformation, pénalité et jeu offensif).

La figure 9, ci-dessous, montre les résultats concernant l'évaluation de l'intensité pour les trois groupes. C'est encore une fois l'essai et le jeu offensif qui sont perçus comme portant le plus d'intensité émotionnelle. Il est intéressant de noter que les groupes $\mathrm{J}$ et FF obtiennent des résultats très similaires dans l'évaluation de l'intensité et c'est le groupe contrôle qui se démarque avec des niveaux d'intensité plus faibles. Il semblerait donc que l'accès au contenu lexical tempère d'une certaine manière la perception de l'intensité émotionnelle. Une analyse linguistique pourrait nous permettre de mieux comprendre les causes de cette différence de perception. Par rapport aux stimuli filtrés, le processus de filtrage altérant le signal, nous avions anticipé des difficultés quant à l'évaluation de l'intensité. Apparemment, le filtrage n'a pas empêché l'évaluation et celle-ci semble cohérente avec l'autre condition (groupe J). Toutefois, on note que la répartition sur l'échelle d'intensité est proche de celle sur l'échelle d'excitation. Il se pourrait qu'il y ait eu une confusion entre excitation et intensité, le jugement d'excitation fourni en premier, influençant l'évaluation de l'intensité. 


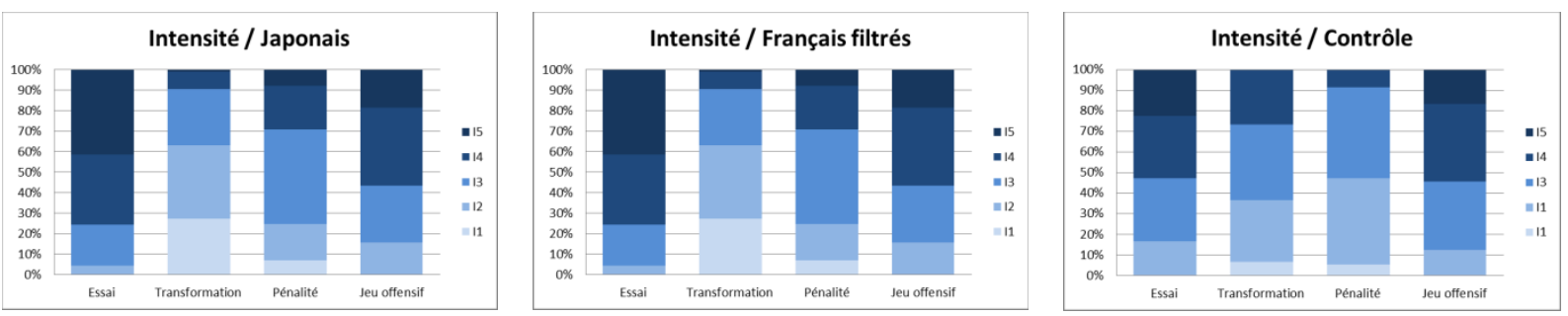

Figure 9 : Evaluation de l'intensité par les trois groupes (groupe J, groupe FF, groupe Contrôle) pour chacun des quatre types d'actions de jeu (essai, transformation, pénalité et jeu offensif).

\subsection{Discussion}

Ce second test de perception en intégrant une échelle supplémentaire, l'excitation, nous a permis de mieux caractériser l'émotion sportive, tout en confirmant les résultats du test précédent. On voit que les actions décisives comme les essais sont bien caractérisés par une intensité et une excitation fortes et une valence positive. Les actions collectives comme le jeu offensif sont également perçues avec des niveaux d'excitation et d'intensité élevés, même si c'est dans une moindre mesure que l'essai ; la valence est également plutôt positive.

Entre les trois conditions d'écoute (stimuli en japonais, stimuli en français et filtrés, stimuli en français), des différences de perception apparaissent naturellement, mais on observe toutefois une forte cohérence entre les jugements des trois groupes. On ne trouve par exemple pas de contradiction complète de perception pour un type d'action d'un groupe à l'autre et sur aucune des trois échelles. Cela laisse à penser que même sans l'accès au lexique une forme d'émotion sportive peut être détectée et caractérisée.

\section{DISCUSSION GENERALE ET CONCLUSION}

Les deux tests de perception que nous venons de présenter avaient pour objectif premier de mieux définir "l'émotion sportive ». Il semble donc que l'émotion sportive soit caractérisée par une intensité forte (c'est un résultat que l'on retrouve dans les deux tests), une excitation importante également (cf. section 4.2.). L'évaluation de la valence est moins facile à définir :

- Le premier test de perception oppose les actions manquées aux actions décisives en terme de valence, et les étiquettes négatives «nerveux » et «agacé » (pour les actions manquées) aux étiquettes positives « heureux » et « content » (pour les actions décisives);

- Le second test tend à montrer plutôt une évaluation positive des actions, y compris des actions qui sont d'une certaine manière des actions manquées comme «le jeu offensif», qui n'a pas permis à l'équipe japonaise de marquer des points, mais qui a renforcé l'effet de suspense sur la fin du match.

Les difficultés d'évaluation de la valence étaient anticipées, surtout sans l'accès à l'information lexicale : d'autres études portant sur l'émotion ont relevé un manque de fiabilité de cette échelle, surtout quand seule l'information prosodique est disponible. On voit bien par ailleurs que c'est sur la valence que le groupe contrôle est le plus en désaccord avec les groupes J et FF.

Si on se base donc sur l'évaluation qui a été faite dans ces deux tests, l'émotion sportive est marquée par un haut degré d'excitation et une intensité forte : en cela, elle pourrait être liée à des émotions telles que la colère ou la joie qui partagent ces mêmes caractéristiques. La valence peut ensuite peut-être nous permettre de faire une différence ou peut-être faut-il accepter que l'émotion sportive est avant tout une forme d'excitation, intense, et que dans certains cas, une valence positive ou négative colore l'expression émotionnelle. Cette coloration serait par ailleurs plutôt portée par le contenu lexical, tandis que la prosodie renseignerait l'excitation et l'intensité. Ces hypothèses pourraient être confirmées par une analyse prosodique qui nous permettrait de corréler les patrons mélodiques bien connus maintenant de la colère et de la joie avec celui/ceux de l'émotion sportive. De même, une analyse lexicale permettrait sans doute de mieux évaluer le poids de cette information dans la perception de la valence positive ou négative de l'émotion.

L'objectif du second test de perception était de déterminer si la prosodie seule permettait de détecter et de caractériser l'émotion sportive. Il semblerait que, comme pour les émotions traditionnelles, la prosodie est une part importante dans l'expression et la perception de l'émotion sportive. Pour ce second test de perception, nous avons testé deux méthodes de masquage de l'information lexicale : le passage par une langue étrangère aux sujets pour empêcher l'accès au lexique ne peut fonctionner que si l'on considère que l'expression de l'émotion sportive à travers les aspects prosodiques présente des caractéristiques universelles, même si par ailleurs les deux systèmes de langue sont relativement éloignés. La cohérence des jugements entre les deux groupes testés (stimuli japonais et français filtrés) laisse à penser qu'il y aurait effectivement des formes universellement reconnues d'expression de l'émotion sportive. 
Enfin, il s'agissait pour les deux tests de vérifier que l'expression de l'émotion sportive était à la fois le résultat d'une contrainte de genre, mais aussi de contenu, et que certaines actions proéminentes étaient plus sûrement destinées à porter de l'émotion. Cette hypothèse s'est vérifiée en partie : ainsi, les essais qui sont les actions de jeu qui marquent le plus de points semblent systématiquement animés d'une forme d'expression émotionnelle. En revanche, les pénalités, qui ont pourtant permis au Japon de marquer beaucoup de points dans cette compétition sont moins marquées du point de vue de l'émotion sportive. Il faut sans doute se placer au niveau pragmatique pour pouvoir expliquer ces différences. Ainsi, la pénalité qui est jouée comme un coup de pied vers l'en-but adverse est une action construite en deux temps: une phase de préparation du tireur relativement longue qui est couverte par du « color-commentary », suivie de la phase de tir proprement dite, très courte, où il s'agit essentiellement pour le journaliste d'énoncer le résultat (le ballon est passé entre les buts ou pas) de l'action et le nouveau score. L'ensemble de l'action, c'est-à-dire les deux phases, a été portée à l'évaluation des sujets : un découpage plus resserré autour de la fin de l'action par exemple aurait peut-être donné une évaluation différente. Ces éléments en tout cas renforcent notre idée que l'image doit être ici prise en compte dans la construction du discours et de l'émotion sportive.

\section{REFERENCES BIBLIOGRAPHIQUES}

Augendre, S., Mathon, C., Boyé, G., Kupsc, A. (2014), « Influence des contraintes extra-linguistiques sur le discours : cas du commentaire sportif télévisé », in Proceedings of CMLF 2014, Berlin, 19-23.

Augendre, S., Kupsc, A., Boyé, G., Mathon, C. (2018), «Live TV sports commentaries: specific syntactic structures and general constraints », in (Legallois, D. Charnois, T., Larjavaara, M., eds.) The Grammar of Genres and Styles: From Discrete to Non-Discrete Units, De Gruyter Mouton, Trends in Linguistics, p. 194.

Bradley, M.M., Lang, P.J., (1994), " Measuring emotion: The self-assessment manikin and the semantic differential », Journal of Behavior Therapy and Experimental Psychiatry 25, p. 49-59.

Mayer. J.D, Gaschke, Y.N., (1988), « The experience and meta-experience of mood », in J Pers Soc Psychol 55, p. $102-111$.

Deulofeu, J. (2000), Les commentaires sportifs constituent-ils un "genre", au sens linguistique du terme ? in Bilger, M. (ed.), Corpus: Méthodologie et applications linguistiques, Paris : Champion.

Fontagnol, C., Hanote, S. et C. Mathon, (2018), « L'émotion dans les commentaires sportifs télévisés en direct : de la production à la perception », Communication au Colloque international La palette des émotions, Poitiers, Octobre 2018. Article accepté pour publication aux Presses Universitaires de Rennes.

Hartmann, C. (2011), Pre-fabricated Speech Formulas as Long-term Memory Solutions to Working Memory Overload. Thèse de doctorat. Université de Zurich.

Lortal, G., Mathon, C., (2008), « Motion and Emotion or how to align emotional cues with game actions », in Proceedings of EMOT Workshop, LREC 2008, Marrakech.

Mathon, C., Boulakia, G. (2009), «Le commentaire sportif en direct: une combinatoire de différentes fonctions de la prosodie. » Interface Discours Prosodie, France.

Mathon, C., Boyé, G., Kupsc, A., (2015), « Contraintes sur le discours et genre de discours contraint : le commentaire sportif télévisé en direct », Discours [En ligne], 17.

Mathon, C., Boyé, G., Kupsc, A., (2016), « Commentaire sportif en direct : Étude des correspondances entre le rythme du jeu et le rythme de parole », in Franck Neveu, Gabriel Bergounioux, Marie-Hélène Côté, Jean-Michel Fournier, Linda Hriba, Sophie Prévost (dir.), Proceedings of CMLF 2016, Tours. https://doi.org/10.1051/shsconf/20162702011.

Niedenthal, P., Dalle, N., (2001), « Le mariage de mon meilleur ami : Emotional response categorization during naturally induced emotions », European Journal of Social Psychology 31, p. 737-742.

\footnotetext{
'Boyé, G., Mathon, C., Petit, M. (2019), Complétude et réduction des noms dans le commentaire sportif. Avec Chabal c'est direct, complet et plein d'émotion., Communication lors du 33e Colloque du Cercle Linguistique du Centre et de l'Ouest (CerLiCO), 24-25 Mai 2019, Bordeaux.

ii https://www.rugbyrama.fr/rugby/coupe-du-monde/2007/live-japon-fidji_mtc163711/live.shtml
} 